
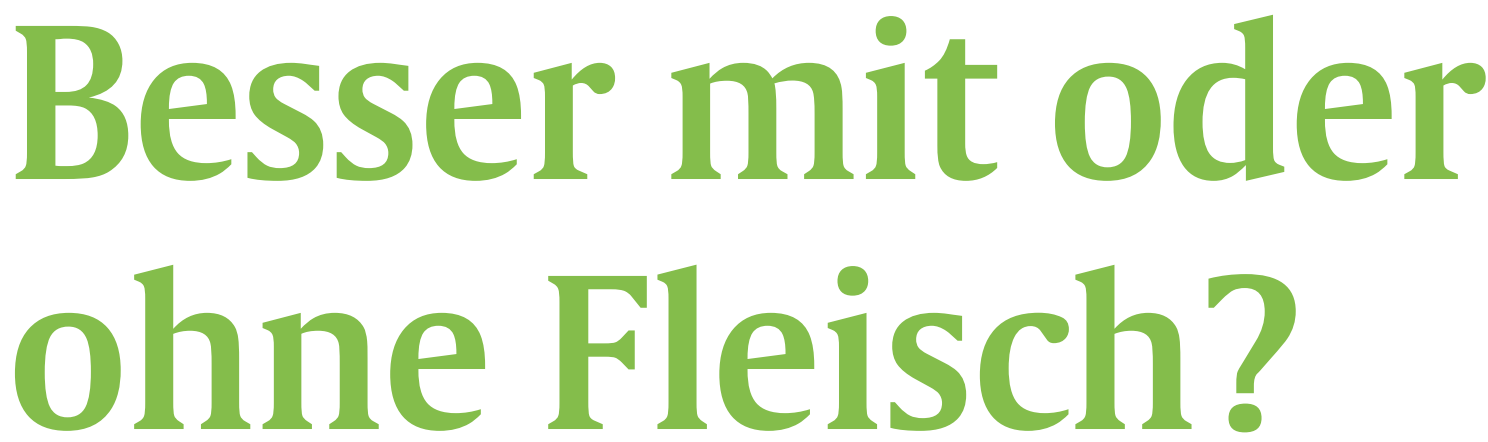

Die Ernährung ist ein wichtiger Bestandteil der integrativen Krebstherapie. Doch welche ist die richtige? Sollten Krebspatienten lieber vegetarisch essen oder eher Fleisch? Oder ist viel Fett bei dieser zehrenden Erkrankung das Beste für den Körper? Unsere Ernährungsexperten erklären, welche Vorteile die vegetarische Kost, die Paläo-Diät und die ketogene Diät bei Krebs haben.
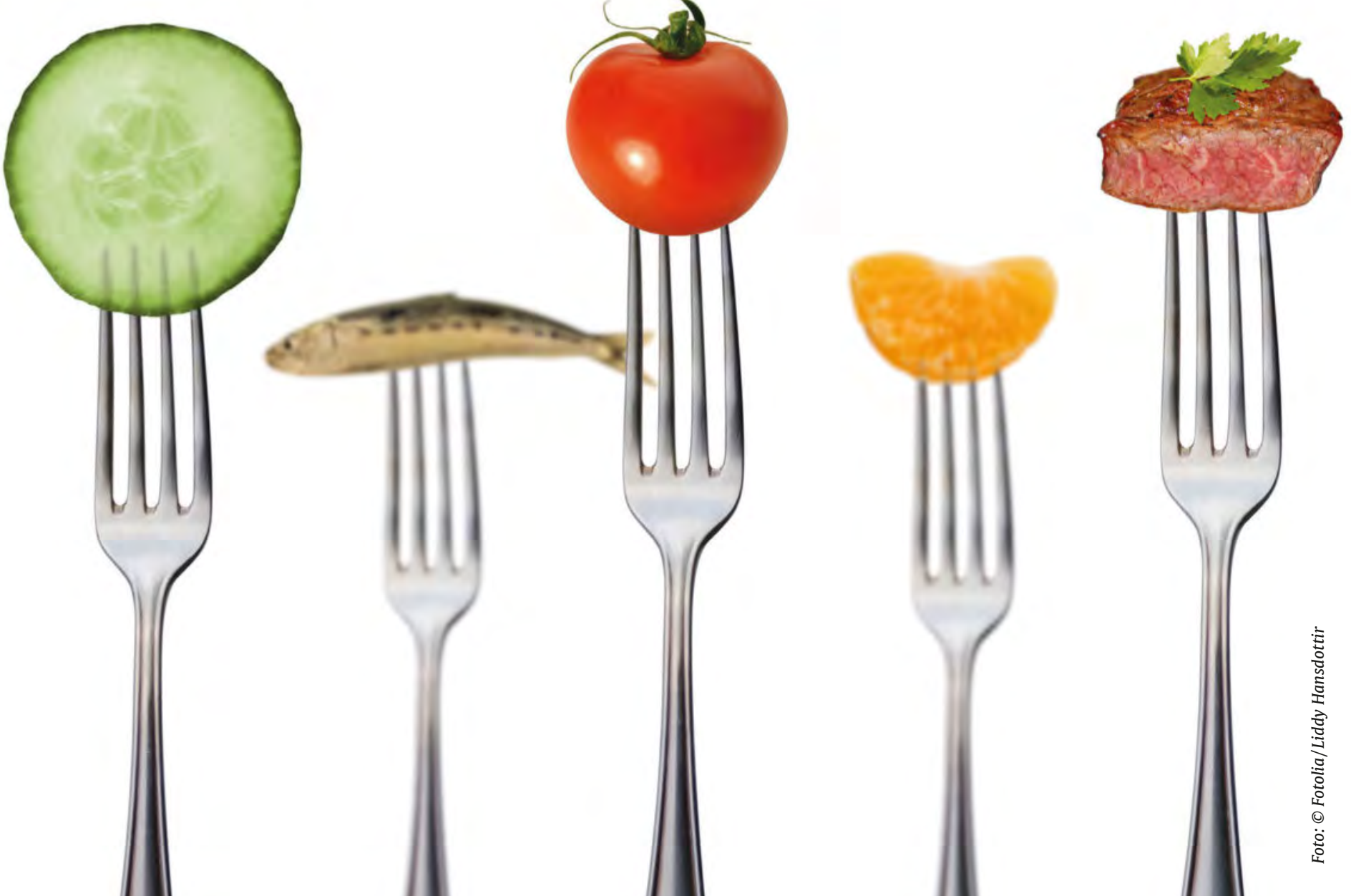


\section{Vegetarische Ernährung bei Krebs}

Claus Leitzmann

Eine Vielzahl wissenschaftlicher Studien zeigt, dass eine vegetarische Ernährung aus gesundheitlichen, ethischen, ökologischen und sozialen Gründen als Dauerkostform empfehlenswert ist [10]. Sie ist vor allem in ihrer Langzeitwirkung für die Prävention und die Therapie verschiedener Krankheiten geeignet [1317]. Dies gilt auch für Krebserkrankungen.

\section{Wie die Ernährung Einfluss nimmt}

Krebs ist wie keine andere Krankheit multikausal bedingt. Endogene Faktoren wie genetische Disposition und Alter sowie exogene Faktoren wie Umwelt, Ernährung, Konsum von Alkohol und Tabak sowie Strahlenbelastung können Einfluss auf die Entstehung von bösartigen Tumoren nehmen. Die meisten dieser Faktoren lassen sich gezielt beeinflussen.

Die wohl umfangreichste Veröffentlichung zu Krebs kommt zu dem Ergebnis, dass sich Krebspatienten während und nach überstandener Erkrankung so ernähren sollten, wie es zur Prävention von Krebs empfehlenswert ist [24]. Studien zeigen, dass sich durch die richtige Lebensmittelauswahl das Kolon- und Magenkrebsrisiko um bis zu 90\%, das Brustkrebsrisiko um $50 \%$ und das Risiko für eine Reihe anderer Krebsarten um mindestens 20\% senken lässt [9, 24]. Zu den Risikofaktoren für die Entstehung von Dickdarmkrebs zählen hoher Verzehr von rotem Fleisch und verarbeiteten Fleischwaren und damit auch hoher Fettverzehr, hochkalorische Ernährung, hoher Alkoholkonsum sowie eine ballaststoffarme Kost [25].
Die pflanzliche Ernährung ist bei Krebs deshalb geeignet, weil sie von allen Kostformen das höchste Gesundheitspotenzial besitzt. Dieses findet sich nicht nur in Form von Vitaminen und Mineralstoffen, die auch in tierischen Lebensmitteln enthalten sind, sondern vornehmlich bei den Ballaststoffen und sekundären Pflanzenstoffen. Beide kommen nur in pflanzlichen Lebensmitteln vor.

\section{Bedeutung von Ballaststoffen}

Ballaststoffe sind Nahrungsbestandteile, die von den Verdauungsenzymen des Menschen nicht oder nur unvollständig abgebaut werden können. Sie üben eine Vielzahl an physiologischen Wirkungen aus, die die Gesundheit beeinflussen können (siehe Tabelle 1).

Die größte prospektive epidemiologische Studie zu Krebs und Ernährung in Europa „European Prospective Investigation into Cancer and Nutrition“ (EPIC) meldete, dass sich die Dickdarmkrebsrate um $40 \%$ senken lasse, wenn die Ballaststoffaufnahme auf $35 \mathrm{~g}$ tgl. erhöht würde. Eine Metaanalyse bestätigte, dass eine hohe Zufuhr an Ballaststoffen, vor allem an Getreideballaststoffen und Vollkornprodukten, mit einem reduzierten Risiko für Darmkrebs verbunden ist [1].

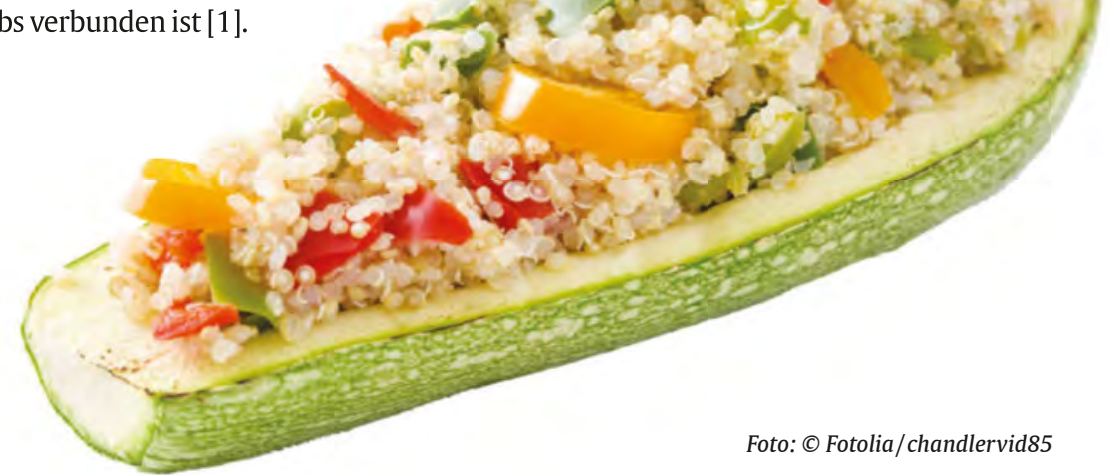

Interventionsstudien, in denen versucht wurde, durch die zusätzliche Gabe von Ballaststoffen (beispielsweise Weizenkleie) bei Patienten mit gutartigen Dickdarmadenomen das erhöhte Risiko für Dickdarmkrebs zu senken, verliefen bislang enttäuschend. Die Gründe liegen wohl in der Reduktion einer gesundheitsfördernden Ernährung auf nur eine ihrer Komponenten - in diesem Fall die Weizenkleie. Ein Isolat wie Kleie wirkt jedoch anders als Ballaststoffe im natürlichen Nahrungsverbund.

\section{Die Krebs-Ballaststoff-Hypothese}

Die in Tabelle 1 genannten Eigenschaften sind Bestandteil der Krebs-BallaststoffHypothese. Sie geht von der Beobachtung aus, dass bei ballaststoffarmer Kost das Auftreten von Dickdarmkrebs erhöht ist [3]. Die Bindung von Gallensäuren an Ballaststoffe zählt zu den möglichen Schutzfunktionen: Sie werden so dem bakteriellen Umbau zu den vermutlich kokanzerogenen sekundären Gallensäu- 
ren entzogen. Ebenso sinkt durch die Erhöhung des Stuhlgewichts und die normalisierte Transitzeit die Verweildauer von schädigenden Substanzen wie biogene Amine und sekundäre Gallensäuren im Darm. Der Kontakt mit der Darmwand reduziert sich. Kurzkettige Fettsäuren, die beim mikrobiellen Abbau von Ballaststoffen im Dickdarm entstehen, tragen zu einem sauren Milieu des Darmlumens bei. Dadurch begünstigen sie das Wachstum wünschenswerter Bakterien, die unerwünschte Fäulnisbakterien verdrängen und die bakterielle Umwandlung von primären in sekundäre Gallensäuren einschränken.
Trotz der bekannten Mechanismen ist der Zusammenhang zwischen Ballaststoffverzehr und Krebsentstehung nicht immer eindeutig. Eine ballaststoffreiche Kost mit Vollkornprodukten, Hülsenfrüchten, Gemüse und Obst ist auch reich an Vitaminen, Mineralstoffen sowie sekundären Pflanzenstoffen. Zudem geht sie oft mit einem geringeren Verzehr von Zucker, Fett und tierischem Eiweiß einher. Deshalb ist ein schützender Effekt hinsichtlich der Entstehung von Darmkrebs aus methodischen Gründen nicht allein auf die Ballaststoffe zurückzuführen.

\section{Bedeutung von sekundären Pflanzenstoffen}

Sekundäre Pflanzenstoffe bestehen aus einer Fülle von Substanzen mit vielen gesundheitsfördernden Eigenschaften (siehe Tabelle 2). Dabei fällt auf, dass alle Subtanzen antikanzerogen wirken. In vielen Fällen ist bekannt, dass sie den Tumorstoffwechsel direkt beeinflussen.

Im Folgenden werden die wichtigsten Erkenntnisse zu den antikanzerogenen Wirkungen dargestellt:

Carotinoide aktivieren Gene, die die Produktion des Proteins Connexin steuern. Es ist Bestandteil von Zellkommunikationsstrukturen. Über sie tauschen die Zellen Signale und Botenstoffe aus, die das Wachstum der Zellen regulieren. In Zel-

\section{Wesentliche Eigenschaften von Ballaststoffen, modifiziert nach [10]}

\begin{tabular}{|c|c|c|c|}
\hline Eigenschaft & primäre Wirkung & sekundäre Effekte & relevante Konsequenzen \\
\hline Faserstruktur & erhöhte Speichelsekretion & frühere und stärkere Sättigungswirkung & bessere Darmgesundheit \\
\hline \multirow{3}{*}{$\begin{array}{l}\text { Wasserbindungsvermö- } \\
\text { gen, Quellfähigkeit, Vis- } \\
\text { kosität }\end{array}$} & $\begin{array}{l}\text { Substrate für bakterielle Fer- } \\
\text { mentation }\end{array}$ & verminderte Gallensäurenresorption & $\begin{array}{l}\text { normalisierte Stuhl- } \\
\text { frequenz }\end{array}$ \\
\hline & $\begin{array}{l}\text { erhöhtes Stuhlgewicht und } \\
\text { Stuhlvolumen }\end{array}$ & bakterielle Bildung kurzkettiger Fettsäuren & $\begin{array}{l}\text { leichteres Absetzen des } \\
\text { Stuhls }\end{array}$ \\
\hline & & normale Transitzeit & \\
\hline \multirow[t]{5}{*}{ Fermentierbarkeit } & $\begin{array}{l}\text { bakterielle Bildung kurzketti- } \\
\text { ger Fettsäuren }\end{array}$ & Senkung des pH-Werts im Kolon & $\begin{array}{l}\text { vermindertes Darmkrebs- } \\
\text { risiko }\end{array}$ \\
\hline & & $\begin{array}{l}\text { positive Wirkung auf qualitative und quantitative } \\
\text { Zusammensetzung der Darmflora }\end{array}$ & \\
\hline & & $\begin{array}{l}\text { Eingeschränkte Bildung von sekundären Gallen- } \\
\text { säuren }\end{array}$ & \\
\hline & & Hemmung der Cholesterinsynthese & \\
\hline & & Wachstum und Differenzierung von Mukosazellen & \\
\hline \multirow[t]{3}{*}{$\begin{array}{l}\text { Adsorptionsfähigkeit, } \\
\text { lonenaustausch }\end{array}$} & Pufferung der Magensäure & verminderte Blutcholesterinspiegel & $\begin{array}{l}\text { verringerte Gallensäuren- } \\
\text { wirkung }\end{array}$ \\
\hline & Bindung von Gallensäuren & verringerte Verfügbarkeit von Schadstoffen & $\begin{array}{l}\text { verminderte Toxizität von } \\
\text { Schadstoffen }\end{array}$ \\
\hline & $\begin{array}{l}\text { Bindung organischer Schad- } \\
\text { stoffe }\end{array}$ & & $\begin{array}{l}\text { vermindertes Darmkrebs- } \\
\text { risiko }\end{array}$ \\
\hline
\end{tabular}


len, die durch Kanzerogene geschädigt wurden, findet dieser Signalaustausch nicht mehr statt. In Anwesenheit von $\alpha-$ und $\beta$-Carotin, Canthaxanthin, Lutein oder Lycopin in der Zellkultur wird die Umwandlung vorgeschädigter Zellen in Krebszellen unterdrückt. Diese Eigenschaft der Carotinoide könnte der Grund für ihren günstigen Einfluss auf die Krebsentwicklung sein. In verschiedenen Studien korrelierte die Carotinoidzufuhr beziehungsweise Carotinoidkonzentration im Blut negativ mit Krebs an Lunge, Prostata, Speiseröhre, Gebärmutterhals, Magen und Dickdarm [11], Brust [2, 7] und Nacken [18].

Phytosterine und ihre Antikrebswirkung wurden bisher nur in vitro und in Tierversuchen untersucht. Beim Menschen müssen die Ergebnisse noch bestätigt werden. Phytosterine scheinen das Krebswachstum in Brust, Prostata, Lunge, Leber, Magen und Eierstock zu vermindern. Als Mechanismen werden diskutiert: die Hemmung der Karzinogenproduktion, das Krebszellwachstum und die Multiplikation, die Invasion und die Metastasierung sowie die Induktion von Zellzyklusstillstand und Apoptose. Die Wirkungsweise einer Krebstherapie unter Verwendung von Phytosterolpräparaten ist noch zu klären [21].

Saponine können das Risiko für Kolonkrebs senken. Sie hemmen die Teilungsrate der Kolonzellen sowie das Wachstum und die DNA-Synthese verschiedener Tumorzellarten. Möglicherweise beruht dieser Mechanismus auf ihrer Fähigkeit, primäre Gallensäuren und Cholesterin zu binden, sodass weniger sekundäre Gallensäuren entstehen. Die chemopräventiven Effekte von Saponinen, Resveratrol und Quercetin sind besonders bei Darmkrebs bekannt [20].

Glucosinolate wie Isothiozyanate und Thiozyanate zeigten in Tiermodellen antikanzerogene Wirkungen in Magen, Brust, Leber und Lunge. Klinische Studien erbrachten, dass sie die Metabolisierung von körpereigenen Östrogenen beeinflussen [8]. Möglicherweise schützen Indole dadurch vor östrogenbezogenen Krebsarten wie Brust- und Endometriumkrebs [5].

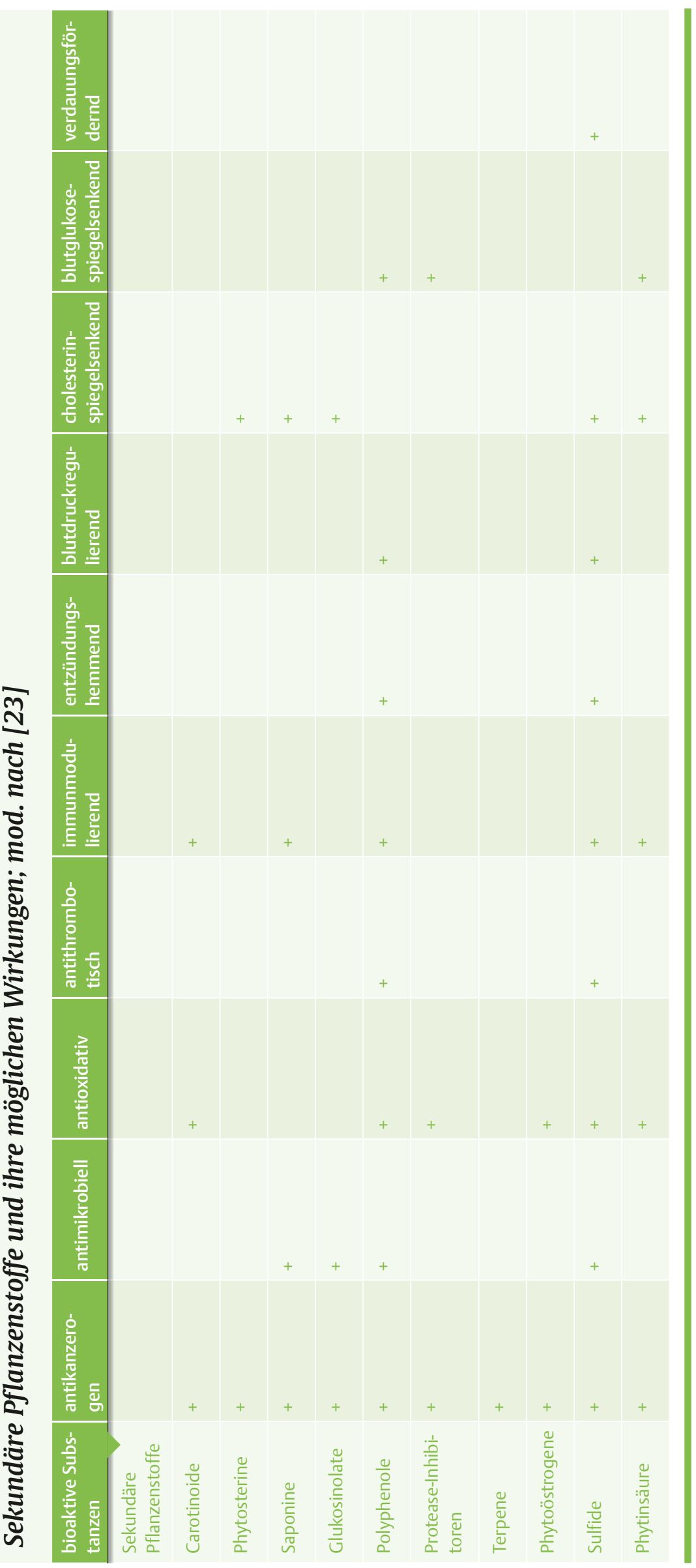


Polyphenole wie Phenolsäuren können vor Krebs an Magen, Speiseröhre, Haut und Lunge schützen [22]. Ihre Wirkung beruht darauf, dass sie Entgiftungsenzyme in Gang setzen, Kanzerogene binden und somit den Kontakt mit der DNA verhindern. Phenolsäuren sind zudem starke Antioxidanzien.

Protease-Inhibitoren und Proteasen sind an Tumorwachstum und Progression beteiligt und gelten als eine wirksame Strategie in der Krebstherapie. Anhand des Typs der Schlüsselaminosäure in der aktiven Stelle der Protease und des Mechanismus der Peptidbindungsspaltung lassen sich Proteasen in Gruppen einteilen [6].

Terpene wie Limonen steigern in Leber und Dünndarm die Aktivität von Entgiftungsenzymen, die in der Tumorprävention Bedeutung erlangen könnten [4].

Phytoöstrogene können als Modulatoren von Östrogenrezeptoren entweder wie Östrogene oder wie Antiöstrogene wirken. Verschiedene Humanstudien zeigen, dass Phytoöstrogene durch ihren Einfluss auf den Hormonstoffwechsel und die Hormonproduktion Schutzpotenzial bei Brustkrebs $[12,19]$ und Kolonkrebs besitzen.

Sulfide verfügen durch ihre antioxidativen und immunstimulierenden Fähigkeiten über antikanzerogenes Potenzial. Zudem beeinflussen sie die Blutgerinnung und wirken verdauungsfördernd, indem sie Speichelfluss, Magensaftsekretion und Darmperistaltik anregen.

Phytinsäure ist für ungünstige Effekte wie die verminderte Resorption verschiedener Mineralstoffe bekannt. Letztlich beeinflusst sie die Gesundheit jedoch günstig: Sie wirkt antikanzerogen sowie regulierend auf den Blutzuckerspiegel.

\section{Fazit}

Der Verzehr einer pflanzlich betonten Kost hat sich bei Krebs bewährt und ist daher empfehlenswert. Die Art der pflanzlichen Ernährung ist von der Dauer und Schwere der Krankheit sowie vom Alter der Betroffenen und der Verträglichkeit der Ernährung abhängig. In keinem Stadium einer Krebserkrankung besteht eine Kontraindikation für die vegetarische Kost. Vielleicht mit einer Aus- nahme: Bei einem starken Verlust an Körpergewicht könnte es erforderlich sein, alle zur Verfügung stehenden Möglichkeiten zu nutzen, um diese Entwicklung aufzuhalten. Hier gilt es, abzuwägen, welcher Kompromiss möglich ist. -

\section{Verwendete Literatur}

[1] Aune D, Chan DS, Lau R et al. Dietary fibre, whole grains, and risk of colorectal cancer: systematic review and dose-response metaanalysis of prospective studies. BM] 2011; 343: d6617

[2] Aune D, Chan DS, Vieira AR et al. Dietary compared with blood concentrations of carotenoids and breast cancer risk: a systematic review and meta-analysis of prospective studies. Am J Clin Nutr 2012; 96: 356-373

[3] Burkitt DP, Walker ARP, Painter NS. Effect of dietary fiber on stools and transit-times, and its role in the causation of disease. Lancet 1972; 2: 1408-1411

[4] Crowell P. Prevention and therapy of cancer by dietary monoterpenes. Nutr 1999; 129 : 775-778

[5] Dinkova-Kostova AT, Kostov RV. Glucosinolates and isothiocyanates in health and disease. Trends Molecular Med 2012; 18: 337-347

[6] Eatemadi A, Aiyelabegan HT, Negahdari B et al. Role of protease and protease inhibitors in cancer pathogenesis and treatment. Biomed Pharmacother 2017; 86: 221-231

[7] Eliassen AH, Liao X, Rosner B et al. Plasma carotenoids and risk of breast cancer over 20 y of follow-up. Am J Clin Nutr 2015; 101 : 1197-1205

[8] Hayes JD, Kelleher MO, Eggleston IM. The cancer chemopreventive actions of phytochemicals derived from glucosinolates. Eur J Nutr 2008; 47(Suppl 2): 73-88

[9] IARC (Internationalen Agentur für Krebsforschung). Welt-Krebs-Bericht. Genf: WHO; 2014

[10] Koerber K v, Männle T, Leitzmann C. Vollwert-Ernährung. Konzeption einer zeitgemäBen und nachhaltigen Ernährungsweise. 11. Aufl. Stuttgart: Haug; 2012

[11] Leenders M, Leufkens AM, Siersema PD et al. Plasma and dietary carotenoids and vitamins A, C and E and risk of colon and rectal cancer in the European Prospective Investigation into Cancer and Nutrition. Int J Cancer 2014; 135: 2930-2939

[12] Leclerq G, Jacquot Y. Interactions of isoflavones and other plant derived estrogens with estrogen receptors for prevention and treatment of breast cancer - considerations concerning related efficacy and safety. J Steroid Biochem Molecular Biol 2014; 139: 237-244

[13] Leitzmann C. Vegetarismus - Grundlagen, Vorteile, Risiken. 4. Aufl. München: Beck; 2012
[14] Leitzmann C. Vegetarismus - Grundlagen, Vorteile, Risiken. München: Beck; 2012

[15] Leitzmann C, Keller M. Vegetarismus. Stuttgart 2013; Ulmer

[16] Leitzmann C. Vegetarismus / Veganismus was dafür spricht. Schweizer Z Ernährungsmed 2015; 12: 15-19

[17] Leitzmann C, Behrendt I. Vegane Ernährung. Erfahrungsheilkunde 2015; 64: 76-83

[18] Leoncini E, Nedovic D, Panic N et al. Carotenoid intake from natural sources and head and neck cancer: A systematic review and meta-analysis of epidemiological studies. Cancer Epidemiol Biomarkers Prev 2015; 24: 1003-1011

[19] Li YH, Niu YB, Sun Y et al. Role of phytochemicals in colorectal cancer prevention. World J Gastroenterol 2015; 21: 9262-9272

[20] Mourouti N, Kontogianni MD, Papavagelis C et al. Diet and breast cancer: a systematic review. Int J Food Sci Nutr 2015; 66: 1-42

[21] Ramprasath VR, Awad AB. Role of phytosterols in cancer prevention and treatment. J AOAC Int 2015; 98(3): 735-738

[22] Roleira FM, Tavares-da-Silva E], Varela CL et al. Plant derived and dietary phenolic antioxidants: anticancer properties. Food Chem 2015; $183: 235-258$

[23] Watzl B, Leitzmann C: Bioaktive Substanzen in Lebensmitteln. 3. Aufl. Stuttgart: Hippokrates; 2005

[24] WCRF / AICR (World Cancer Research Fund/American Institute for Cancer Research). Food, nutrition and the prevention of cancer: a global perspective. Washington: AICR; 2007

[25] WHO (Weltgesundheitsorganisation). Welt-Krebs-Bericht. Genf: WHO; 2014

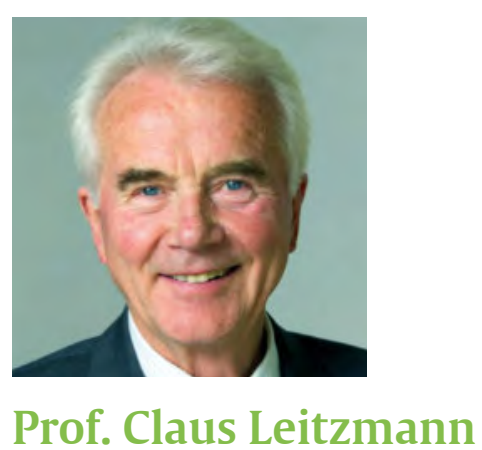

Email: claus@leitzmann-giessen.de Claus Leitzmann, Jahrgang 1933, studierte in den USA. Nach Stationen an der UCLA und Thailand arbeitete er von 1974-1998 am Institut für Ernährungswissenschaft der Universität Gießen. Seine Forschungsgebiete sind die Internationale Ernährung, Vollwert-Ernährung, Ernährungsökologie, Vegetarismus und Nachhaltigkeit. 


\section{Paläo-Ernährung}

bei Krebs

wryour

Paläo- oder Steinzeit-Ernährung hat Konjunktur: Je mehr Studien zeigen, wie positiv sich diese Ernährungsform auf den Körper auswirkt, beispielsweise beim nachhaltigen Abnehmen, desto mehr Anhänger findet sie.

\section{Das Rätsel der Zivilisations- krankheiten}

Die Altsteinzeit, das Paläolithikum, reicht vom Beginn der Menschheit vor ca. 2,5 Millionen Jahren bis zu 10000 Jahren vor unserer Zeitrechnung, also bis zum Beginn der Viehzucht und des Ackerbaus. Erst mit dem Ackerbau, dem Meso- und dem Neolithikum sind chronische dege-

\section{Woher kommt Paläo?}

Der Paläo-Trend (engl. Paleo) hat sich in den letzten 10-15 Jahren in den USA entwickelt. Auslöser dafür war die Zunahme chronischer Erkrankungen. Inzwischen erfreut sich die Paläo-Ernährung großer Resonanz sowohl bei Laien als auch bei Heilpraktikern, funktional arbeitenden Ärzten und Therapeuten. Gerade bei Zivilisationskrankheiten hat Paläo eindeutige therapeutische Qualitäten.

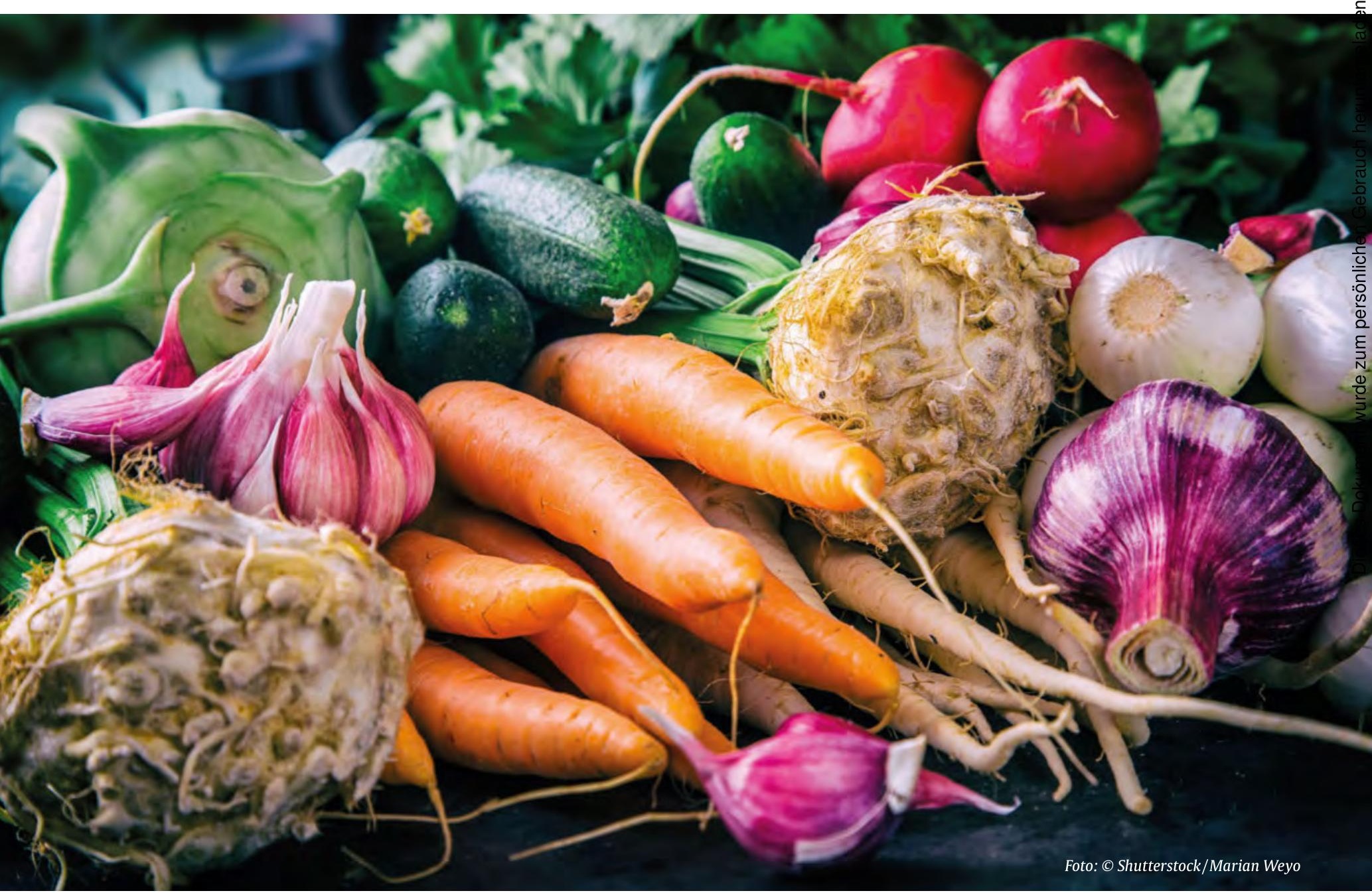


nerative Krankheiten entstanden. Aber, so lautet ein häufiger Einwand, hat das nicht etwas mit unserer Altersentwicklung zu tun? Oder anders gefragt: Sind Menschen in der Altsteinzeit nicht so jung gestorben, dass sie Krebs oder Demenz gar nicht entwickeln konnten? Die Antwort ist: Nein. Steinzeitmenschen starben in jungen Jahren häufig an akuten Infektionen oder einer Sepsis nach Verwundung im Kampf oder Unfällen. Sonst konnten sie durchaus 80 Jahre erreichen. Und das ohne wesentliche degenerative Anzeichen. In Stämmen in Afrika, Südamerika und der Südsee, die heute noch wie ihre altsteinzeitlichen Vorfahren als Jäger und Sammler leben, finden sich praktisch keine Krankheiten wie

\section{- INFORMATION}

\section{Was is(s)t Paläo?}

- Die Paläo-Ernährung orientiert sich am verfügbaren Nahrungsangebot der Vor-Ackerbau-Zeit [5]. Konsequente Paläo-Anhänger jagen sogar noch selbst und essen nur regional und saisonal.

- Gemeinsam ist allen Paläo-Anhängern der möglichst vollständige Verzicht auf industrialisierte Lebensmittel. Vereinfacht gesagt: auf alles, was eine Zutatenliste hat.

- Außerdem fallen industriell raffinierte Öle wie Sonnenblumen-, Rapsöl oder andere Fette weg, die nur industriell bearbeitet essbar sind.

" mindestens $1 \mathrm{~kg}$ pestizidfreies, variantenreiches Gemüse tgl., gedünstet in gesundem Fett oder, wenn Rohkost, als Smoothie

- Fleisch gehört für die meisten Paläo-Anhänger auf den Speiseplan. Jedoch nur von Tieren, die wild gefangen oder ohne Einsatz von Kraftfutter und Medikamenten aufgezogen worden sind. Es ist üblich, Fleisch „from head to tail“ zu essen, inklusive der nährstoffdichten Innereien, Haut und Knochen, deren in Knochenbrühe ausgelöstes Kollagen für die Darmgesundheit einen wichtigen Beitrag leistet.

- Isolierte, schnell anflutende Zucker sowie Getreide und Milch entfallen komplett.

- Lebensmittel wie Hülsenfrüchte, fermentierte Milchprodukte (Käse, Joghurt), Nachtschattengewächse und bei Unverträglichkeiten auch Eier oder Nüsse sind mindestens im therapeutischen Kontext vorübergehend oder dauerhaft zu meiden.
Diabetes, Herz-Kreislauf-Krankheiten oder Krebs. Auch autoimmune Prozesse sind dort weitgehend unbekannt.

\section{Zucker und die Suche nach Ersatz}

In der Praxis bleibt das größte Problem bei der Ernährungsumstellung der Zucker. Erfahrungsgemäß vergeht keine Woche, in der nicht mindestens ein Kunde anfragt, was mit diesem oder jenem Zuckerersatzstoff sei. In diesem Bereich gibt es viele Irrtümer, weil Substanzen wie Stevia oder Xylitol als unbedenklich gelten, was sie schon wegen der von ihnen ausgehenden Suchtbegünstigung nicht sind. Besonders schwer zu verstehen ist für viele der Verzicht auf freie Fruktose, die vor allem in der Kombination mit Omega-6-Fetten Endotoxine bildet und Studien zufolge erheblich zu Übergewicht beiträgt. Die maximal tolerierbare tägliche Menge an Fruktose, gebunden an frisches Obst oder Gemüse, beträgt lediglich ca. $10 \mathrm{~g}$. Das entspricht dem Gegenwert eines Apfels.

\section{Paläo: mehr als Ernährung}

Paläo setzt als Lebensstil obendrein auf ein gezieltes Stressmanagement, mehr Bewegung im Alltag, gesunden Schlaf sowie seltene Mahlzeiten: idealerweise nicht mehr als zwei oder drei am Tag. Alternativ funktionieren auch „Essensfenster" von maximal acht Stunden am Tag, so dass man täglich oder mehrmals wöchentlich intermittierend zwischen 16 und bis zu 36 Stunden fastet. Bis dahin funktioniert die reinigende Apoptose. Erst danach würde der einsetzende „Überlebensmodus“ die Fastenvorteile zunichtemachen.

Intermittierendes Fasten funktioniert jedoch nur, wenn der körpereigene Fettstoffwechsel gut funktioniert, weil der Körper dann übergangslos und ohne Hunger von Fett-Außen- auf Fett-Innenernährung umschaltet. Bei der Ernährungsumstellung ist jedoch immer zu berücksichtigen, dass das keineswegs ein selbstverständlicher Vorgang ist. Die
Mehrheit der Menschen hat heute einen dysfunktionalen Fettstoffwechsel. Hier ist es wichtig, die Verdauung vor allem am Anfang mit Enzymen, Chlorwasserstoff $(\mathrm{HCl})$ und Öl aus mittelkettigen Triglyzeriden (C8) zu unterstützen.

\section{Erfahrungen aus der Praxis}

Der Astrophysiker Paul und die Biochemikerin Shou-Ching Jaminet haben über sieben Jahre die wissenschaftlichen Grundlagen der Paläo-Ernährung zusammengetragen und kritisch überprüft. In meinem Team setze ich die Jaminet'schen Erkenntnisse seit fünf Jahren sehr erfolgreich in der Zusammenarbeit mit hausärztlichen Praxen und Heilpraktikern ein. Die meisten der Patienten haben vorher ein Eiweißprofil (Proteomis-Profil nach C.E.I.A.) [2] erstellen lassen. Dieses zeigt in Kombination mit einer Nahrungsanamnese und einer Bioimpedanzmessung der Körperzusammensetzung, wo die größten Probleme liegen und welche Änderungen über die Umstellung auf Paläo hinaus notwendig sind.

\section{Paläo in der Krebsprävention}

Krebs gilt heute zunehmend wieder als metabolische Krankheit [4]. Hohe Zucker- und Insulinspiegel verschärfen nachweisbar entzündliche Vorgänge, ebenso wie industrialisierte Nahrungsmittel. So setze ich schon im Ernährungsberatungsalltag, nach dem Eiweißprofil nach C.E.I.A., präventiv auf eine individuell optimierte Paläo-Ernährung. Bei Profilen, die hohe Entzündungswerte ausweisen, hat es sich als wirkungsvoll erwiesen, den relativ geringen Kohlenhydratanteil, den eine Paläo-Ernährung ohnehin hat, weiter zu verringern. PaläoAnhänger setzen meistens auf eine Ernährung, deren Kohlenhydratanteil deutlich unter dem der Empfehlungen der DGE liegt, also statt bei $50-60 \%$ bei deutlich unter 30\%.

Eine solche Kohlenhydratbegrenzung bezeichnen Gegner im Sinne eines Warnhinweises gerne als „low carb“. Low Carb im ursprünglichen Sinne bedeutet jedoch 
eine ketogene Ernährung, bei der der Brennstoff des Körpers nahezu ausschließlich aus Ketonen besteht. Das ist dauerhaft nur zu erreichen, wenn sowohl der Kohlenhydratanteil als auch der Proteinanteil deutlich unter $10 \%$ der jeweils täglich zugeführten Kalorien liegen. Davon kann bei einer „normalen“ Paläo-Ernährung keine Rede sein.

Als vorbeugend sehe ich eine Ernährung an, die in einer giftiger werdenden Umwelt möglichst auf zusätzliche Entzündungsimpulse aus Zucker, industriellen Zusatzstoffen und Omega-6-Fetten verzichtet. Nicht umsonst helfen eine Paläo-Ernährung und ein entsprechender Lebensstil in der Regel relativ schnell, sich aus einer diabetischen Stoffwechsellage zu verabschieden, die ja mit einem deutlich erhöhten Krebsrisiko verbunden ist. Für mich ist vor diesem Hintergrund plausibel und erfahrbar, dass Paläo-Ernährung sinnvoll in der Prävention ist nicht nur von Krebs, sondern von allen chronischen und degenerativen Erkrankungen.

\section{Paläo in der Krebstherapie}

Wie aber sieht es bei manifestem Krebs aus? Begünstigt eine Ernährungsweise, die bei ansonsten mehr oder weniger Gesunden unweigerlich zu Abnehmerfolgen führt, nicht die gefürchtete Tumorkachexie? Die Antwort darauf muss differenziert ausfallen: Der Tumor ist ein Zuckerverstoffwechsler, er kann keine Ketone verarbeiten. Es liegt daher nah, die Ernährung auf ein ketogenes Paläo-Protokoll umzustellen, also mindestens vorübergehend auf die extreme Low-CarbVariante von Paläo.

Irrig wäre jedoch die Annahme, dass das allein den Tumor aushungert, da viele Tumore die Fähigkeit haben, sich eine eigene metabolische Versorgung aufzubauen. Es gibt inzwischen jedoch eine Reihe von Studien und viele Fallberichte, die zeigen, dass eine ketogene Ernährung mindestens den Fortgang der Krebserkrankung hemmt.

Um einen möglichen Gewichtsverlust qualitativ beurteilen zu können, ist es hilfreich, nicht nur eine Waage einzusetzen, sondern die Körperzusammensetzung und die mitochondriale Kraftstoffversorgung durch eine Bioimpedanzmessung zu ermitteln. Obendrein sollte der Bauchumfang bestimmt werden. Meist zeigt sich dann, dass ein Gewichtsverlust aufgrund der Ernährungsumstellung keine Kachexie begünstigt. Es kommt zu einer Gewichtsabnahme ohne Verlust der Magermasse: Der Körper wird nicht ausgehungert, sondern entlastet.

Nicht zuletzt zeigt sich, dass Menschen in einer Nahrungsketose Chemound Strahlentherapie deutlich besser überstehen. Es gibt damit keinen Grund, bei Krebs auf eine nährstoffdichte, energetisch fettbetonte Paläo-nahe Ernährung zu verzichten.

Sehr häufig wenden Therapeuten jedoch ein, man würde damit dem Krebskranken „den letzten Rest an Lebensfreude nehmen“. Natürlich ist der Zuckerentzug einer der härtesten Entzüge überhaupt. Das gilt übrigens auch für Menschen, die nicht eine solche akute Motivation wie Krebs haben. Doch abgesehen davon, dass es hierfür eine ganze Reihe an konkreten Hilfestellungen gibt, käme wohl niemand auf die Idee, einem Raucher, der an Lungenkrebs erkrankt ist, zu erlauben oder gar zu empfehlen weiterzurauchen. Dabei wird seine Lebensqualität durch den Nikotinentzug zweifellos zunächst beeinträchtigt.

Als Fazit lässt sich ziehen, dass PaläoErnährung nach der sich ständig verdichtenden Studienlage und nach meinen Erfahrungen sicher keine hinreichende, wohl aber eine notwendige Antwort ist. Und zwar nicht nur auf Krebs [1, 6].

\section{Verwendete Literatur}

[1] Ballantyne, S. The Paleo Approach. Reverse Autoimmune Disease and Heal Your Body, Las Vegas: Victory Belt Publishing; 2013

[2] Fischer S. Hrsg. Funktionelle Proteomik. Krankheitsursachen frühzeitig erkennen und gezielt behandeln. München: Urban \& Fischer Verlag/Elsevier; 2008

[3] Jaminet P, Jaminet S-J. Perfect Health Diet. Regain Health and Lose Weight by Eating the Way You Were Meant to Eat. New York: Scribe UK; 2012
[4] Seyfried, T. Cancer as a Metabolic Disease. On the Origin, Management, and Prevention of Cancer. Hoboken: John Wiley \& Sons; 2012

[5] Shanahan C. Deep Nutrition. Why Your Genes Need Traditional Food. 1. Aufl. New York: St Martin's Press; 2016

[6] Wahls T. The Wahls Protocol. How I beat progressive MS Using Paleo Principles and functional medicine. New York: Avery; 2014

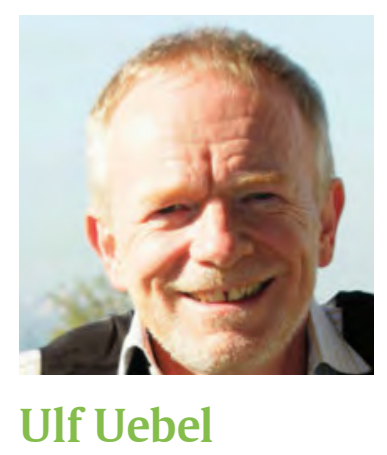

E-Mail: u.uebel@health21.de

Ulf Uebel, MA, arbeitet als Health-Coach (Certified Transformational Nutrition Coach, Certified Gluten Practitioner) in ganz Deutschland mit Ärzten und Heilpraktikern zusammen. Dabei setzt er vor allem auf individuelle Ernährungsumstellung auf der Basis des Proteomis-Profils (funktionelles Eiweißprofil nach C.E.I.A.). 


\section{Ketogene Ernährung in der Krebstherapie}

Ulrike Kämmerer und Rainer Johannes Klement

Ketogene Diäten (KDs) sind sehr fettreiche, stark kohlenhydratreduzierte Ernährungsformen mit bedarfsgerechter Eiweißversorgung. Sie liefern in der Regel mindestens 75\% der Energie aus Fett und maximal 5-10\% aus Kohlenhydraten (KH). KDs sind in verschiedenen Varianten beschrieben als „Atkins-Diät“, „klassische ketogene Diät“ oder auch als „LowCarb (LC) Diät“.
Die häufig verwendeten eiweißlastigen, aber relativ fettarmen LC-Formen stellen keine echte KD dar. Denn sie sind nicht mit einer zuverlässigen systemischen Ketose verbunden, die als Leitsymptom der KD gilt. Eine stabile Ketose ist nur bei niedrigen Insulinspiegeln und damit bei Nahrungskarenz oder einer sehr fettreichen und eiweiß-/ kohlenhydratarmen Ernährung zu erreichen.

\section{Ketogene Diäten bei Krebs}

KDs können bei Krebserkrankungen unterstützend wirken. Der veränderte Stoffwechsel der Patienten ist durch eine gesteigerte Fettoxidation bei gleichzeitiger peripherer Insulinresistenz gekennzeichnet [1] und spricht dadurch gut auf den hohen Fettanteil der Ernährung an. Der reduzierte KH-Anteil hält den Blutzucker und den Wachstumsfaktor Insulin

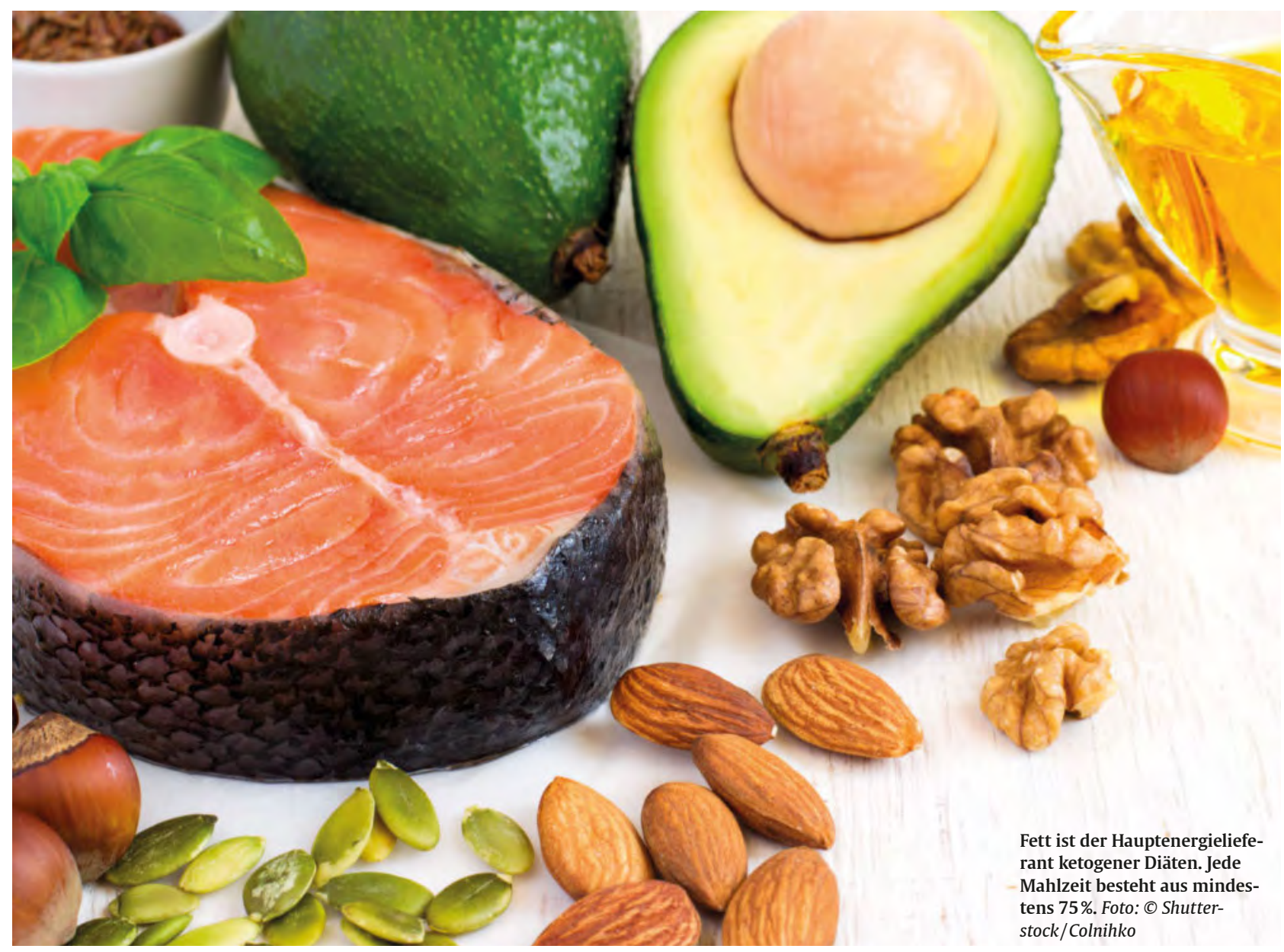


niedrig. Ein „Aushungern von Krebs“ durch Zuckerentzug ist jedoch auch mit einer KD nicht möglich - der Blutzucker bleibt auf Nüchternniveau stabil. Durch die geringere Aufnahme von Glukose, dem entscheidenden Ausgangssubstrat zum Aufbau neuer Tumorzellen, ist vor allem in schlecht durchbluteten Tumorarealen ein langsameres Wachstum durch Tierstudien bestätigt [2, 3]. Daten der Grundlagenforschung der letzten Jahre zeigen zudem, dass gerade $\beta$-Hydroxybutyrat (3-OHB) wichtige, das Tumorzellwachstum stimulierende Signalproteine hemmen kann und sich auch als HDAC-Inhibitor erwiesen hat $[2,4]$.

\section{Möglichkeiten und Grenzen}

Bei der Übertragung von Daten aus Zellkultur oder Mäusen auf den Menschen ist jedoch Vorsicht geboten. Mäuse besitzen neben einer unterschiedlichen Insulinund Blutzuckerregulation [5] einen etwa 7-fach höheren Ruheenergiestoffwechsel als Menschen. Deshalb reagieren sie schneller und drastischer auf Kalorien-

- INFORMATION

\section{Systemische Ketose oder Ketoazidose?}

Bei der systemischen Ketose kommt es zu erhöhten Spiegeln der physiologischen Ketonkörper Acetoacetat (AcAc) und $\beta$-Hydroxybutyrat (3-OHB). 3-OHB wird dabei im Verhältnis von 1:1 bis 3:1 zu AcAc gebildet. In der Ketose steigen die Serumspiegel von nüchtern 0,10,2 mM auf 1-6 mM an. Der Blutzucker bleibt bei einer physiologischen Ketose auf Nüchternniveau, Blut-pH und Elektrolyte befinden sich im Normbereich, und die Betroffenen sind fit und wach. Das sind wesentliche Unterschiede zur potenziell lebensbedrohlichen Ketoazidose, die bei relativem oder absolutem Insulinmangel, beispielsweise bei Diabetikern und Alkoholikern auftreten kann. Eine Ketoazidose ist durch eine arterielle Azidose ( $\mathrm{pH}<7,35)$, einen sehr hohen Blutzuckerwert (über $250 \mathrm{mg} / \mathrm{dl}$ ) sowie ein Basendefizit gekennzeichnet. Der Patient ist schläfrig bis komatös. oder Makronährstoffentzug [6]. Im Gegensatz zu den Tiermodellen konnten klinische Studien bisher keine lebensverlängernden Effekte einer ausschließlichen KD zeigen [7-9]. Manche Daten deuten auf eine leichte Hemmung des Tumorglukosestoffwechsels durch eine fettreiche und kohlenhydratarme Ernährung hin [10-12]. Es erscheint nach derzeitigem Wissensstand dennoch unwahrscheinlich, mit einer KD alleine das Tumorwachstum sicher aufhalten zu können.

Vielversprechender ist ein unterstützender Ansatz, in dem eine KD beispielsweise mit Strahlen- und Chemotherapie kombiniert wird [13]. Schon eine leichte Hemmung der Glykolyse würde für die Tumorzelle eine Reduktion ihrer antioxidativen Abwehr bedeuten, was sie sensibler gegenüber freien (Sauerstoff-)Radikalen (ROS) macht. Diese entstehen zum Beispiel durch die Radiolyse von Wasser während der Bestrahlung.

Eine verstärkte Wirkung von Strahlen-, Chemo- und antiangiogener Therapie durch KDs wurde in mehreren Tiermodellen belegt und deutet sich auch in klinischen Daten von Glioblastompatienten an [9]. Bei den ersten fünf Tumorpatienten, die in der Strahlentherapie Schweinfurt mit kurativer Strahlentherapie und KD behandelt wurden [14], zeigt sich auch nach gut zwei Jahren noch kein Hinweis auf ein Lokalrezidiv. Der mögliche Beitrag der KD zu diesem Ergebnis bleibt allerdings spekulativ. Ein unmittelbarer Nutzen der ketogenen Ernährung war jedoch in einer Verbesserung der Lebensqualität und dem Erhalt fettfreier Masse während der Therapie gegeben.

Ein weiterer interessanter Aspekt ergibt sich durch ein kurzzeitiges Fasten (24-48 h) vor einem Chemotherapiezyklus, um Nebenwirkungen zu vermindern und dabei die Anti-Tumor-Wirkung zu erhalten oder sogar zu verstärken [15]. Die Reduktion von Glukose und Insulin bei gleichzeitiger Erhöhung der Ketonkörper soll gesunde Zellen, jedoch nicht Tumorzellen vor zelltoxischen Angriffen schützen. In zwei klinischen Studien zeigten sich bisher in der Tat positive Effekte des Fastens vor der Chemotherapie $[16,17]$. Das weist auf eine Bedeutung

\section{HDAC-Inhibitoren in der Krebs- therapie}

HDAC steht für Histon-Deacetylasen. Es handelt sich um Enzyme, die unter anderem den Zellzyklus kontrollieren. Sie sind wichtige Zielmoleküle, die von modernen Krebsmedikamenten gezielt gehemmt werden - mit durchaus nachgewiesenen klinischen Effekten.

der Ketonkörper hin und würde somit auch für eine KD als ,fastenimitierende Diät" sprechen, falls kurzzeitiges Fasten kontraindiziert ist [18]. Auch wäre der Ansatz wieder, die KD mit einer zytotoxischen Therapie zu kombinieren.

\section{Fazit und Umsetzung}

Die ketogene Ernährung ist aus theoretischer Sicht für die Krebsbehandlung interessant, und zeigt in den meisten Tierstudien günstige Effekte auf Tumorwachstum und Überleben. Die bisherigen Daten am Menschen weisen darauf hin, dass ihre Stärke vor allem in der Kombination mit Standardtherapien zu sehen ist. Bisher konnte keine Studie negative Effekte einer KD auf den Allgemeinzustand der Patienten oder ihre Erkrankung feststellen. Daher sollte der ketogenen Ernährung während der Krebstherapie eine Chance eingeräumt werden. Dies gilt vor allem begleitend zu kurativen Ansätzen. Allerdings können sich auch in der Palliativtherapie positive Effekte auf die Körperzusammensetzung oder Lebensqualität zeigen. Voraussetzung für eine wirksame KD ist neben der Motivation der Betroffenen eine fachkundige Ernährungsberatung und Betreuung durch Ärzte oder Heilpraktiker.

Für die ketogene Diät geeignete und ungeeignete Lebensmittel: siehe Tabellen in der Online-Version.

http://dx.doi.org/10.1055/s-0043-112664 


\section{Verwendete Literatur}

[1] Barber MD, McMillan DC, Preston T et al. Metabolic response to feeding in weight-losing pancreatic cancer patients and its modulation by a fish-oil-enriched nutritional supplement. Clin Sci 2000; 98(4): 389-399

[2] Shukla SK, Gebregiworgis T, Purohit V et al. Metabolic reprogramming induced by ketone bodies diminishes pancreatic cancer cachexia. Cancer Metab 2014; 2: 18

[3] Klement R], Champ CE, Otto C et al. Anti-Tumor Effects of Ketogenic Diets in Mice: A Meta-Analysis. PLoS One 2016; 11:e0155050

[4] Rojas-Morales P, Tapia E, Pedraza-Chaverri ]. $\beta$-Hydroxybytate: A signaling metabolite in starvation response? Cell Signal 2016; 28 : 917-923

[5] Chandrasekera PC, Pippin J]. Of Rodents and Men: Species-Specific Glucose Regulation and Type 2 Diabetes Research. ALTEX 2013; 31: 157-176

[6] Mahoney LB, Denny CA, Seyfried TN. Calorie restriction in C57BL/6] mice mimics therapeutic fasting in humans. Lipids Health Dis 2006; 5: 13

[7] Schmidt M, Pfetzer N, Schwab M et al. Effects of a ketogenic diet on the quality of life in 16 patients with advanced cancer: A pilot trial. Nutr Metab 2011; 8: 54

[8] Rieger ], Bähr O, Maurer GD et al. ERGO: A pilot study of ketogenic diet in recurrent glioblastoma. Int J Oncol 2014; 44: 1843 1852

[9] Schwartz K, Chang HT, Nikolai M et al. Treatment of glioma patients with ketogenic diets: report of two cases treated with an IRB-approved energy-restricted ketogenic diet protocol and review of the literature. Cancer Metab 2015; 3: 3

[10] Bozzetti F, Gavazzi C, Mariani L et al. Glucose-based total parenteral nutrition does not stimulate glucose uptake by humans tumours. Clin Nutr 2004; 23: 417-421

[11] Fine E], Segal-isaacson C], Feinman RD et al. Targeting insulin inhibition as a metabolic therapy in advanced cancer: A pilot safety and feasibility dietary trial in 10 patients. Nutrition 2012; 28: 1028-1035

[12] Schroeder U, Himpe B, Pries R et al. Decline of lactate in tumor tissue after ketogenic diet: in vivo microdialysis study in patients with head and neck cancer. Nutr Cancer 2013; 65: 843-849

[13] Klement R], Champ CE. Calories, carbohydrates, and cancer therapy with radiation: Exploiting the five R's through dietary manipulation. Cancer Metastasis Rev 2014; 33: 217-229

[14] Klement RJ, Sweeney R. Impact of a ketogenic diet intervention during radiotherapy on body composition: I. Initial clinical experience with six prospectively studied patients. BMC Res Notes 2016; 9: 143
[15] Brandhorst S, Harputlugil E, Mitchell JR et al. Protective effects of short-term dietary restriction in surgical stress and chemotherapy. Ageing Res Rev 2017 Feb 20. pii: S15681637(17)30033-8

[16] de Groot S, Vreeswijk MP, Welters M] et al. The effects of short-term fasting on tolerance to (neo) adjuvant chemotherapy in HER2-negative breast cancer patients: a randomized pilot study. BMC Cancer 2015; 15: 652

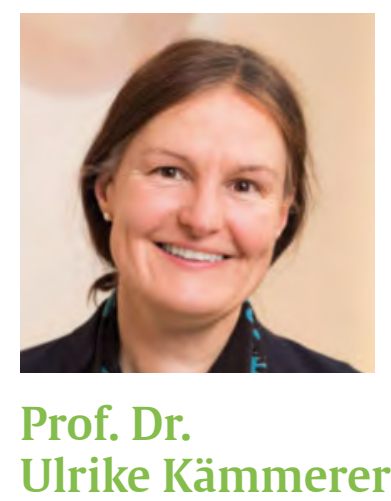

Frauenklinik und Poliklinik Josef-Schneider-Str. 4, Haus C15 97080 Würzburg

E-Mail: u.kaemmerer@uni-wuerzburg.de

Prof. Dr. Ulrike Kämmerer studierte Diplom-Biologie in Erlangen und promovierte über virusausgelöste Herzinsuffizienz. Seit 1996 ist sie an der Frauenklinik Würzburg als Wissenschaftlerin in der Grundlagenforschung Immunologie (Habilitation über Reproduktionsimmunologie) und Stoffwechsel tätig. Seit 2008 ist sie für die fachliche Betreuung klinischer Studien rund um das Thema ketogene Diät und Ernährung bei Krebserkrankungen zuständig.
[17] Dorff TB, Groshen S, Garcia A et al. Safety and feasibility of fasting in combination with platinum-based chemotherapy. BMC Cancer 2016; 16: 360

[18] Klement RJ. Fasten als Nebenwirkungsmanagement? InFo Onkol 2016; 19: 22-24

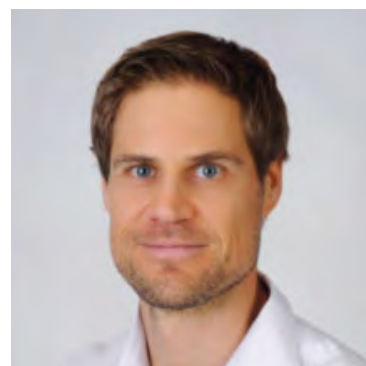

\section{Dr. Rainer Johannes Klement}

Leopoldina Krankenhaus Schweinfurt Klinik für Strahlentherapie und Radioonkologie

Gustav-Adolf-Str. 6-8

97422 Schweinfurt

E-Mail: rainer_klement@gmx.de

Dr. Rainer Johannes Klement studierte Physik in Heidelberg und promovierte am Max-Planck-Institut für Astronomie, wo er anschließend noch zwei Jahre als wissenschaftlicher Mitarbeiter tätig war. Danach wechselte er in die Medizinphysik an die Universität Würzburg, wo er begann, sich mit Fragen der Strahlenbiologie und des Tumorstoffwechsels und deren Beeinflussung durch diätetische Maßnahmen zu beschäftigen. Seit 2013 arbeitet und forscht Dr. Klement in der Strahlentherapie Schweinfurt, wo derzeit die KETOCOMP Studie zur ketogenen Ernährung unter seiner Leitung läuft.

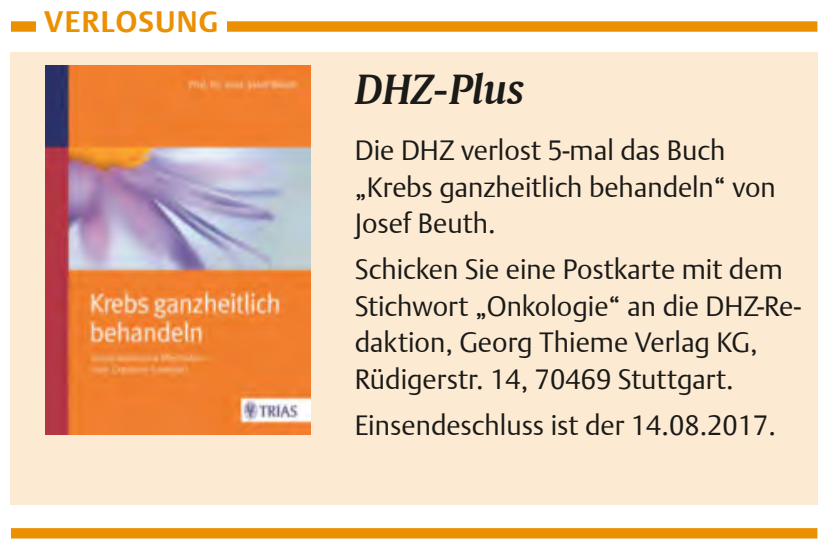


- TABELLE 1

\section{Gut geeignete Lebensmittel}

\begin{tabular}{|c|c|}
\hline & Gemüse \\
\hline Sehr gut & $\begin{array}{l}\text { Artischocke, Aubergine, Bambussprossen, Staudensellerie, Blumenkohl, Brokkoli, Chinakohl, Fenchel, Grünkohl, Gurken, } \\
\text { Knollensellerie, Mangold, Paprika, Portulak, Radieschen, Rettich, Rhabarber, Sauerkraut, Schwarzwurzeln, Spargel, Spinat, } \\
\text { Tomate, Wirsing, Zucchini }\end{array}$ \\
\hline \multirow[t]{2}{*}{ Mäßig gut } & $\begin{array}{l}\text { Bohnen (grün), Kohlrabi, Kürbis, Möhren, Lauch, Petersilienwurzel, Rosenkohl, Rotkohl, Steckrübe, Topinambur, weiße } \\
\text { Rüben, Weißkraut }\end{array}$ \\
\hline & Obst (natur, ungezuckert) \\
\hline Mäßig gut & $\begin{array}{l}\text { Acerola, Brombeeren, Erdbeeren, Guave, Heidelbeeren, Himbeeren, Holunderbeeren, Johannisbeeren, Moosbeeren, Papa- } \\
\text { ya, Preiselbeeren }\end{array}$ \\
\hline \multirow[t]{2}{*}{ perfekt } & Avocado \\
\hline & Nüsse und Samen \\
\hline \multirow[t]{2}{*}{ Sehr gut } & Hanfnüsse, Kokosnuss, Leinsamen, Macadamianüsse, Mandeln, Mohnsamen, Paranüsse, Pekanüsse \\
\hline & Milch und Milchprodukte \\
\hline Sehr gut & $\begin{array}{l}\text { Butter, Crème fraîche (40\%), Dickmilch, Frischkäse (Doppelrahm), Naturjoghurt (10\%), Käse (ideal mind. } 60 \%) \text {, Mascarpo- } \\
\text { ne, Schlagsahne (mind. } 30 \% \text { ), saure Sahne (10\%), Schafmilch, Schmand (24\%), Speisequark (40\%), Tofu, Ziegenmilch }\end{array}$ \\
\hline \multirow[t]{2}{*}{ Mäßig gut } & Buttermilch, Hafermilch, Mandelmilch, Schmelzkäse, Scheibletten, Sojamilch, Stutenmilch \\
\hline & Salate, Pilze, Antipasti, Kräuter, Sprossen, Hülsenfrüchte \\
\hline Sehr gut & $\begin{array}{l}\text { Bohnensprossen, Eisbergsalat, Endiviensalat, Feldsalat, Frühlingszwiebel, Gartenkresse, Kopfsalat, Oliven (grün und } \\
\text { schwarz), Radicchio, Rucola, Sauerampfer, Schnittlauch, alle Pilze bis auf Shiitake und Trüffel }\end{array}$ \\
\hline \multirow[t]{2}{*}{ Mäßig gut } & Ingwer, Meerrettich, Petersilie, Shiitake, Trüffel \\
\hline & Fleisch, Fisch und Eier \\
\hline Sehr gut & Bacon, Bratwürste, Eier, fettes Fleisch (ideal aus Weidehaltung), fette Meeresfische, fette Streichwurst \\
\hline Mäßig gut & magere Fische, mageres Fleisch (ideal aus Weidehaltung) \\
\hline
\end{tabular}


- TABELLE 2

\section{Weniger geeignete Lebensmittel}

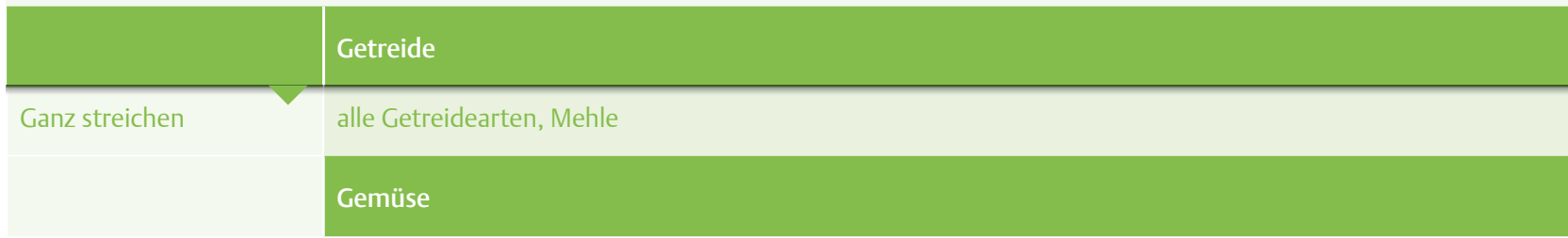

Ganz streichen

Kartoffeln, Rote Bete, Süßkartoffeln

Obst, Trockenobst, Fruchtsäfte

Nur sehr gelegentlich in

Ananas, Apfel, Birne, Feige, Grapefruit, Honigmelone, Kaktusfeige, Kirschen (süß und sauer), Kiwi, Mandari-

kleinen Mengen nen, Mango, Maulbeere, Mispel, Nektarine, Passionsfrucht, Pfirsich, Pflaumen, Reineclaude, Wassermelone

Ganz streichen

Bananen, Datteln, Ebereschenbeere, Granatapfel, Hagebutten, Kaki, Kumquat, Litchi, Mirabellen, Weintrauben, alle Sorten von Trockenobst einschließlich Weintrauben/Sultaninen, alle Fruchtsäfte, Fruchtsmoothies

Nüsse und Samen

Nur sehr gelegentlich in kleinen Mengen

Cashewkerne, Erdnüsse, Haselnüsse, Kastanien (Maronen), Kürbiskerne, Pinienkerne, Pistazienkerne, Sesamsamen, Sonnenblumenkerne, Walnüsse

Milch und Milchprodukte

Ganz streichen

Milch, Milchprodukte mit Fruchtzubereitung, „Schokoladengeschmack“, „Vanille“ und andere süße Geschmacksrichtungen

Salate, Pilze, Antipasti, Kräuter, Sprossen, Hülsenfrüchte

Bohnen (Kerne, alle Sorten), Erbsen, Kichererbsen, Linsen

Fertiggerichte

Ganz streichen

Pizza, Pasta, Döner usw. 